\title{
Ancoragem esquelética com mini-implantes
}

\author{
Skeletal Anchorage with mini-implants
}

\author{
*Réferson M. dos Santos ${ }^{1}$ \\ Rafaela S. Barbosa M. dos Santos² \\ Daniela Avila Mól Avena
}

\section{Resumo}

A utilização de dispositivos de ancoragem como coadjuvante no tratamento ortodôntico, tem demonstrado, nos últimos anos, alta versatilidade de aplicação clínica, principalmente no que tange o uso dos mini-implantes. A ancoragem pode ser definida em Ortodontia como a resistência ao movimento dentário indesejado e poder ser obtida tanto por mecanismos intrabucais como por meio de aparelhos extrabucais, o que torna a colaboração do paciente imprescindível para o sucesso do tratamento. O objetivo deste trabalho é avaliar os diversos aspectos referentes aos mini-implantes ortodônticos e suas aplicações clínicas, procurando justificar que a ancoragem em ortodontia é assunto de grande relevância no planejamento ortodôntico.

\section{Palavras-chave}

Ancoragem esquelética; Mini-implantes; Aplicações clínicas.

\footnotetext{
1 Doutor em Implantodontia pela Faculdade São Leopoldo mandic, Campinas-SP, Mestre em Cirurgia e Traumatologia Bucomaxilofacial pela Pontifícia Universidade Católoca do Rio Grande do Sul, Graduado em odontologia pela Universidade Federal da Bahia (UFBA), Especialista em Radiologia Bucomaxilofacial pelo CEBEO-Bahia, Professor de Cirurgia da Faculdade de Tecnologia e Ciências, campus Salvador (FTC) e Universidade Estadual de Feira de Santana (UEFS).

2 Graduada em odontologia pela faculdade de Tecnologia e Ciências FTC ,Salvador-Ba e Especialista em Ortodontia pela Faculdade de Tecnologia e Ciências FTC, Salvador-Ba.

${ }^{3}$ Graduada pela Faculdade de Odontologia Unifenas-MG, Especialista em Ortodontia pela Faculdade de Tecnologia e Ciências e Especialista em Implantodontia pelo CEBEO-Bahia.
} 


\begin{abstract}
The use of devices for anchoring as an adjuvant in orthodontic treatment, has demonstrated, in recent years, high versatility of clinical application, especially in the use of the mini-implants. The anchorage can be set in orthodontics as the resistance to motion unwanted dental and can be achieved either by mechanisms intrabucais as through extrabucais apparatus, which makes the patient's cooperation is essential for the success of treatment. The objective of this study is to evaluate the various aspects relating to the mini-implants orthodontic and its clinical applications, seeking to justify that the anchorage in orthodontics is a matter of great relevance in planning orthodontic.
\end{abstract}

\title{
Keywords
}

skeletal Anchorage; mini-implants; clinical applications. 


\section{INTRODUÇÃO}

A ancoragem esquelética revolucionou os conceitos dos tratamentos ortodônticos, proporcionando movimentação dentária com minimização dos efeitos indesejáveis na unidade de reação. (VILLELA et al.,2006). Visando reduzir a necessidade de colaboração dos pacientes e ampliar as possibilidades de tratamento, alguns tipos de implantes têm sido utilizados como auxiliares do tratamento ortodôntico, fornecendo a possibilidade de uma ancoragem absoluta (VILLELA et al.,2008).

Existem muitos recursos de ancoragem como arco extraoral, barra transpalatina, arco de Nance, porém apresentam desvantagens que vão desde a pouca eficiência do método escolhido à necessidade de cooperação pelo paciente. Os aparelhos extrabucais têm sido utilizados para obtenção de uma ancoragem eficaz, podendo orientar o crescimento em pacientes jovens, assim como eliminar o perigo de movimentação dentária indesejada, causada por forças recíprocas intermaxilares (KLOEHN, 1947). A ancoragem intrabucal é vastamente utilizada e pode ser classificada em intramaxilar (consiste na utilização de grupos de dentes dentro do mesmo arco e que se opõem à movimentação de dentes individuais ou de grupos menores) ou intermaxilar (utiliza a arcada oposta como apoio para transmissão de força, podendo a direção dessas forças variar conforme as necessidades clínicas e tendo em vista que movimentações dentárias indesejadas podem ocorrer na arcada de ancoragem (PROFFIT et al.,1995).

\section{Histórico dos mini-implntes}

GAINSFORTH e HIGLEY (1945) foram os primeiros a considerarem possível a utilização de implantes como ancoragem ortodôntica na movimentação dentária, utilizando fios ortodônticos e parafusos metálicos de vitálio cirúrgico, fixado ao osso na região da mandíbula de um cão.

Desde 1965, quando Branemark e colaboradores descreveram a osseointegraçao de 1969 quando relataram a utilização de parafusos de titânio osseointegraveis como ancoragem intra-óssea para prótese total, mudanças significantes ocorreram em todas as áreas da Medicina. Na Odontologia, a partir deste evento, definiu-se a especialidade da Implantologia, que hoje, com o avanço das pesquisas, tornou-se uma grande aliada da Ortodontia.
Kanomi (1997) foi o primeiro a publicar um sistema de mini-implantes com desenho específico para uso na ortodontia, descrevendo um protocolo cirúrgico de um implante com medidades de $1,2 \mathrm{~mm}$ de diâmetro por $6 \mathrm{~mm}$ de comprimento.

Segundo Costa et al.,1998, foram os primeiros a descreverem a técnica no uso de mini-implantes com extremidades externa com ranhuras retangulares, simulando o encaixe de um braquete e sugeriram vários sítios de instalação.

Atualmente os mini-implantes ortodônticos de titânio tem sido utilizados como importantes métodos de ancoragem em Ortodontia, servindo de apoio em diversos tipos de movimentos dentários, considerados difíceis ou complexos para os métodos tradicionais de ancoragem como, por exemplo, casos assimétricos e de intrusão de molares.

\section{Mini-Implantes}

Na busca por um recurso de ancoragem esquelética mais versátil, percebeu-se que os parafusos de titânio para fixação cirúrgica, apesar de seu tamanho reduzido, possuíam resistência suficiente para suportar a maioria das forças ortodônticas (KYUNG et al., 2003; PARK et al., 2002). Os dispositivos de ancoragem podem ser classificados como extrabucais ou intrabucais. A escolha depende das necessidades clínicas apresentadas e mediante uma análise criteriosa e individualizada dos objetivos do tratamento.

Segundo NOJIMA (2006), os mini-implantes são em sua maioria, confeccionados de liga de titânio. Variam quanto à forma, design e medidas, de acordo com a marca comercial. Possuem três porções distintas: cabeça (área para instalação de dispositivos ortodônticos), porção transmucosa ou pescoço (região existente entre a porção rosqueável e a cabeça do implante) e porção rosqueável (parte ativa do mini-implante).

Os mini-implantes ainda podem ser classificados em autoperfurantes, onde não existe a necessidade de perfuração prévia na maioria dos casos e os autorosqueáveis que adota a perfuração prévia apenas da cortical óssea. Aqueles utilizados nos últimos anos apresentam variações de diâmetro (1.0, 1.2, 1.4, 1.6, $1.8 \mathrm{e}$ $2.0 \mathrm{~mm})$, altura (4-15mm) e confeccionados em titânio (CHENG et al., 2004; GRAY; SMITH, 2000; KANOMI; TAKADA, 2000;). A seleção das partes do mini-implante deve ser feita conforme a necessidade individual de cada caso, para se adaptar às condições anatômicas 
juntamente com as necessidades ortodônticas para o sucesso do tratamento (VILLELA et al., 2008).

$O$ conceito de ancoragem esquelética não é novo, pois há 60 anos já havia sido sugerida a ancoragem no osso basal como alternativa para aumentar a ancoragem convencional (LEE et al., 2007). Com a utilização dos implantes, surge uma nova alternativa de ancoragem esquelética em Ortodontia, a qual não permite a movimentação da unidade de reação.

\section{Aplicações Clínicas}

Os mini-implantes apresentam diversas indicações clínicas, entre elas: retração do segmento anterior, evitando o uso de aparelho extrabucal como reforço de ancoragem, verticalização de molares, intrusão de dentes anteriores e até mesmo intrusão de molares. Apesar do pequeno diâmetro (variações de 1 a $2 \mathrm{~mm}$ ), os mini-implantes são capazes de suportar forças de até $450 \mathrm{~g}$, enquanto a maioria das forças utilizadas em Ortodontia é inferior a 250g (KYUNG et al., 2003).

\section{Intrusão de Molares e Incisivos}

A intrusão de molares é considerada um movimento ortodôntico demasiadamente difícil e complexo de se realizar usando métodos tradicionais de ancoragem. Existem, no entanto, situações clínicas nas quais este movimento se faz necessário para obtenção de um bom resultado no tratamento, como, por exemplo, na extrusão de molares causada pela perda de dentes antagonistas (VILLELA et al., 2006).

As intrusões dentárias podem ser realizadas por meio do movimento de translação ou de inclinação. Quando o movimento de intrusão pura é necessário, deve-se planejar a passagem da linha de ação de força através do centro de resistência. No plano transversal, para se conseguir a intrusão pura deve-se instalar duas forças, uma por vestibular e outra por palatino para produzir uma força resultante que passe próximo do centro de resistência. No plano sagital para se conseguir intrusão de corpo deve-se instalar duas forças, uma por mesial e outra por distal (Figura 1). Quando o movimento de intrusão com inclinação é necessário, a linha de ação de força deverá passar mais distante do centro de resistência, com intuito de gerar um momento (tendência de rotação).

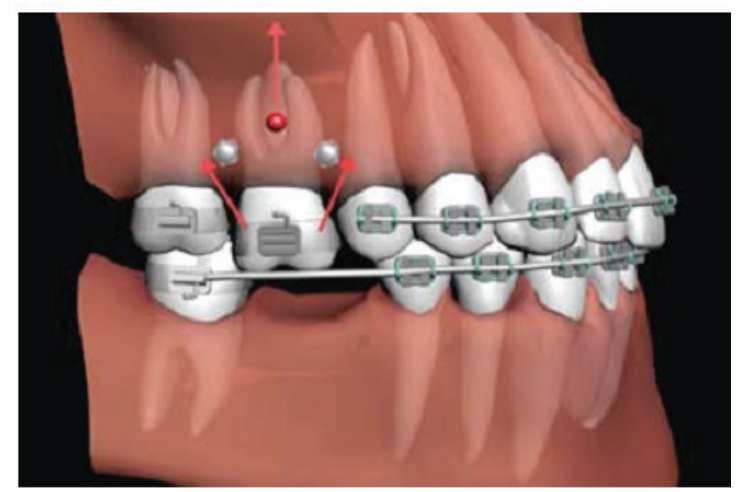

Figura 1: Utilizaçãomini-implantes promovendo a intrusão com controle axial

(VILLELA et al.,2008)

Em relação a intrusão de incisivos superiores, a posição ideal para instalação dos mini-implantes depende da inclinação destes dentes. Quando se apresentam verticalmente dispostos ou retroinclinados, recomenda-se utilizar um único mini-implante na linha média, o mais alto possível, próximo a espinha nasal anterior (COSTA et al.,1998). Já para intrusão dos incisivos inferiores (Figuras 2 e 3), também verticalizados e retroinclinados, o mini-implante deve ser posicionado entre os centrais, o mais baixo possível (CARANO et a 2005; COSTA et al.,1998; KANOMI, 1997).

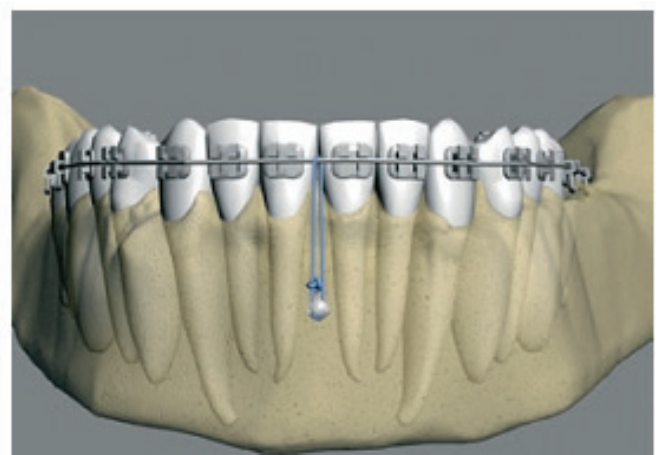

Figuras 2 e 3: Intrusão de incisivos superiores e inferiores com intuito de promover inclinação para vestibular (ARAÚJO et al., 2008) 
Quando os incisivos estão com boa inclinação axial (Figuras 4 e 5), deve-se fazer com que a linha de ação de força passe o mais próximo possível de centro de resistência dos dentes que serão movimen-

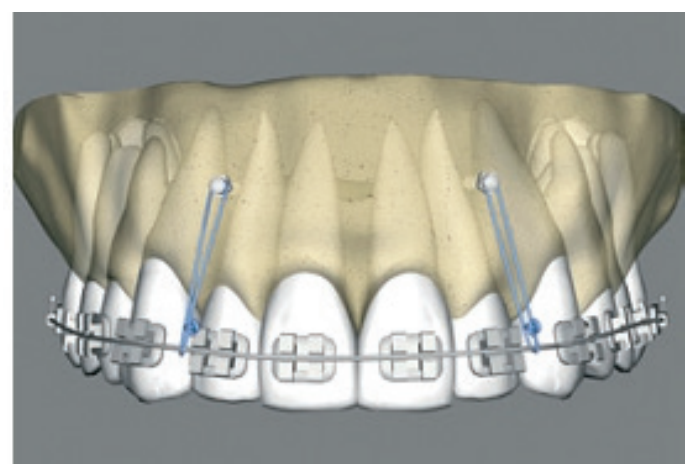

tados. Sendo assim, sugere-se utilizar dois mini-implantes, um de cada lado, posicionados entre os incisivos laterais e caninos (CARANO et al.,2005; KANOMI, 1997).

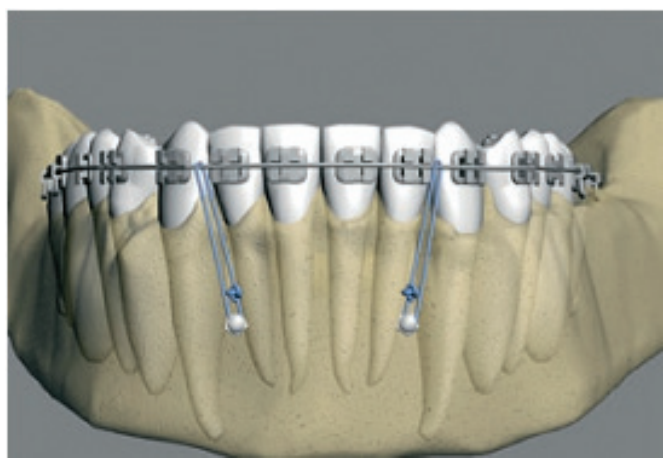

Figuras 4 e 5: Intrusão de incisivos superiores e inferiores com intuito de manter a inclinação axial dos dentes (ARAÚJO et al., 2008)

\section{Mesialização de Molares}

A mesialização de molares com ancoragem esquelética consiste no movimento mesial destes dentes sem que haja reações nos segmentos mais anteriores do arco. $\mathrm{O}$ fechamento dos espaços de primeiros molares perdidos precocemente deve ser realizado com movimento de corpo dos segundos molares e, às vezes, também dos terceiros, o que significa deslocamento anterior que varia de 12 a $15 \mathrm{~mm}$ (JANSON, 2008).

Dentre as indicações destacam-se o fechamento de espaços edêntulos decorrente de perda dos dentes permanentes, o fechamento de espaços devido a agenesias de dentes posteriores, especialmente os pré-molares e em casos de extração de dentes permanentes de prognóstico duvidoso (JANSON et al.,2001).

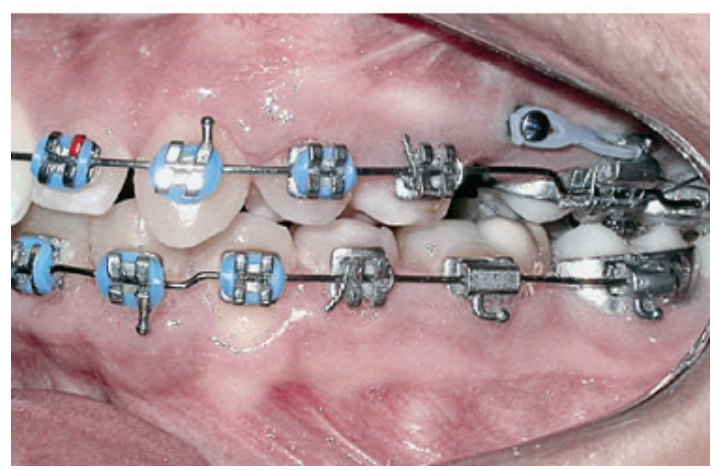

Figura 6: Foto lateral esquerda durante a mesialização do molar (JANSON, 2008)

\section{Distalização de Molares}

A distalização de molares consiste numa excelente estratégia de tratamento para correção das más oclusões de Classe II de natureza dentoalveolar ou para compensação das más oclusões de Classe II de natureza esquelética moderada. Com a utilização dos mini-implantes como unidade de ancoragem, as distalizações são executadas eficientemente, sem efeitos colaterais indesejados (VILLELA et al., 2008).

Em pacientes adultos ou adolescentes, onde há indicação do uso de aparelho extrabucal para distalizar molares, os mini-implantes ortodônticos podem ser utilizados como substitutos de forma eficiente, funcional e estético (BEZERRA et al., 2004; VILLELA et al., 2004). A figura 7 mostra um cursor conectado ao mini-implante por meio de uma mola de NITI e a força aplicada no cursor foi transferida ao primeiro molar.

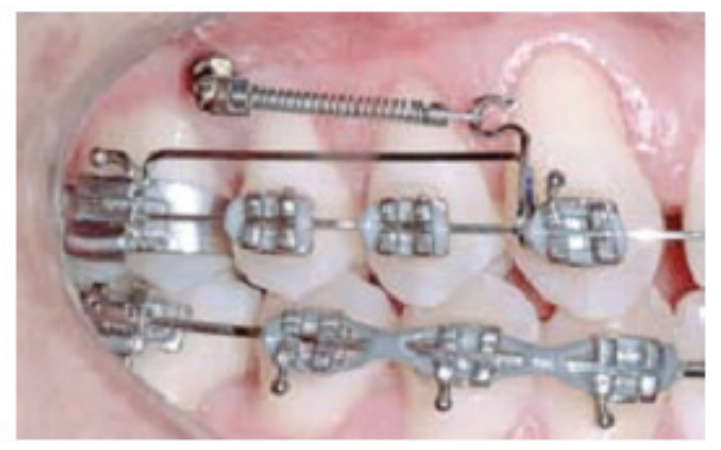

Figura 7. Cursor conectado ao mini-implante (VILLELA et al.,2008) 


\section{DISCUSSÃO}

Os mini-implantes fazem parte da mecânica ortodôntica contemporânea, apresentando taxas de sucesso cada vez mais altas. De acordo com KELES et al. (2004) a utilização dos mini implantes para ancoragem esquelética tem demonstrado ser uma modalidade de tratamento eficiente, não só para um procedimento de rotina na ortodontia, mas também para resoluções de casos mais complexos ou difíceis na odontologia.

De acordo com MARASSI et al. (2011), este é um novo recurso que vem como uma opção a mais no tratamento ortodôntico a fim de simplificar a mecânica em alguns casos reduzindo os números de acessórios colocados nos dentes, com menor tempo de tratamento e boa aceitação do paciente. A própria técnica de instalação apresenta forte influencia sobre o resultado final. Dentre os fatores relacionados ao ato cirúrgico que influenciam positivamentedestacam-se: experiência do operador, estabilidade primária, perfuração previa, ausência de vibrações e torque final de inserção (CHADDAD et al., 2008; LM et al.2008).

\section{CONSIDERAÇÕES FINAIS}

Apesar da possibilidade de utilização de diversos métodos convencionais de ancoragem intra ou extrabucais, intra ou intermaxilares, a utilização de mini-implates possibilita a obtenção de um sistema de ancoragem, eficaz e com grande aplicabilidade clínica.

Sua simplicidade de instalação e remoção predispõe a uma grande aceitação e conforto por parte do paciente e torna a mecânica ortodôntica mais efetiva, por meio do maior controle da unidade de ancoragem, sem a presença de movimentos recíprocos indesejáveis. O sucesso deste recurso de ancoragem, porém, depende de cuidados que passam por detaIhado planejamento ortodôntico, aplicação de forças e manutenção da saúde Peri-implantar.

\section{REFERÊNCIAS}

1. Bezerra F, Laboissière JR. M, Villela H, Diaz L. Ancoragem Ortodôntica Absoluta Utilizando Micro-Parafusos de Titânio: Planejamento e Protocolo Cirúrgico. Implant News, São Paulo, v.1, n.5, p. 33-39. 2004

2. CARANO, A.; VELO, S.; LEONE, P.; SICILIANI, G. Clinical applications of the miniscrew anchorage system. J. Clin. Orthod., Boulder, v. 39, no. 1, p. 9-24, Jan. 2005.

3. COSTA, A.; RAFFAINL, M.; MELSEN, B. Miniscrews as orthodontic anchorage: a preliminary report. Int. J. Adult. Orthodon. Orthognath. Surg., Chicago, v. 13, no. 3, p.201-209, 1998

4. CHADDAD, K., FERREIRA, A. H., GEURS, N. Influence of surface characteristics Angle on survival rates of mini-implants. Orthodontic., v. 78, n.1, 107-13, 2008.

5. Cheng, S.J.; Tseng I. Y.; Lee J.J.; Kok S.H.: A prospective study of the risk factors associated with failure of mini implants used for orthodontic anchorage. Int Oral Maxilofac Implants 2004;19:100-106.

6. Gray, J.B., Smith R. Transitional implants for orthodontic anchorage. J Clin Orthod. V. 34, n. 11, p. 659-666, 2000.

7. JANSON, M. Verticalização de molares. In: __. Ortodontia em adultos e tratamento interdisciplinar. Maringá: Revista Dental Press de Ortodontia e Ortopedia Facial. Maringá, 2008. p. 129-167.

8. JANSON, M. R. P.; JANSON, R. R. P.; MARTINS, P. F. Tratamento interdisciplinar I: considerações clínicas e biológicas na verticalização de molares. Revista Dental Press de Ortodontia e Ortopedia Facial. Maringá, v. 6, no. 3, p. 87-104, 2001

9. Kanomi, R.: Mini-implant for orthodontic anchorage. Int J Clin Orthod,31:763-767. Nov.1997.

10. KYUNG, S. H.; HONG, S. G.; PARK, Y. C. Distalization of maxillary molars with a midpalatal mini-screw. J clin Orthod, Boulder, v. 37, no. 1, p. 22-26, Jan. 2003.

11. Kanomi R, Takada K (2000). Application of titanium mini- implant system for orthodontic anchorage [M]// Davidovitch Z, Mah J. Biological mechanism of tooth movement and craniofacial adaption. Birmingham (AI): EBSCO Media; pp. 253-258.

12. Keles A, Everdi N, Sezen S. Bodily distalization of molars with absolute anchorage. Angle Orthod. 2003;73(4):471-82. 
13. LEE, J. S., KIM, J. K., PARK, Y. Applications of orthodontic mini-implants Canadá: Quintessense, 2007.

14. LIM, S., CHA, J., HWANG, C. Insertion torque of orthodontic miniscrews according to changes in shape diameter and lengh Angle. Orthodontic., v. 78, n. 2, p. 234-40, 2008.

15. Marassi C, Leal A, Herdy JL. Clinical applications of mini screws as anchorage. In: American Association of Orthodontist 104Th Annual Session, Florida: AAO, 2004.

16. NOJIMA, L. I. et al. Dispositivos temporários de ancoragem em Ortodontia. In: BERNARDES, J. Estética em Implantologia. 1. ed. São Paulo: Quintessence, 2006.

17. Proffit, W.R; ROHDE, A.C; SHELDEN, F.C. Fundamentals of anchorage, force and movement. Am. J. Orthod. St Louis, v. 34, n. 10, p. 860-867, Oct. 1995.

18. VILLELA H, VILLELA P, BEZERRA F, SOARES AP, LABOISSIÈRE JR. M. Utilização de mini-implantes para anco- ragem ortodôntica direta. Innovations Journal, v. 8, n.1, p. 05-12. 2004

19. VILLELA, H. M.; BEZERRA, F.J.B.; LEMOS, L.N.; PESSOA. Intrusão de molares superiores utilizando microparafusos ortodônticos de titânio autoperfurantes. Revista Clínica de Ortodontia Dental Press. Maringá, v. 7, n. 2, abr-mai,p. 52-64, 2008.

20. VILLELA, H.; BEZERRA, F.; LABOISSIERE E JUNIOR, M. Microparafuso ortodôntico de titânio auto-perfurante: novo protocolo cirúrgico e atuais perspectivas clinicas. Innovations Implant Journal, São Paulo, v.1, n. 1, p. 46-53, 2006.

21. VILLELA, H.; BEZERRA, F.; MENEZES, P.; VILLELA, F.; LABOISSIERE JUNIOR, M. Microparafusos ortodônticos de titanio auto-perfurantes: mudando os paradigmas da ancoragem esquelética em Ortodontia. Implant news. São Paulo, v. 3, n. 4, p. 45-51, 2006. 